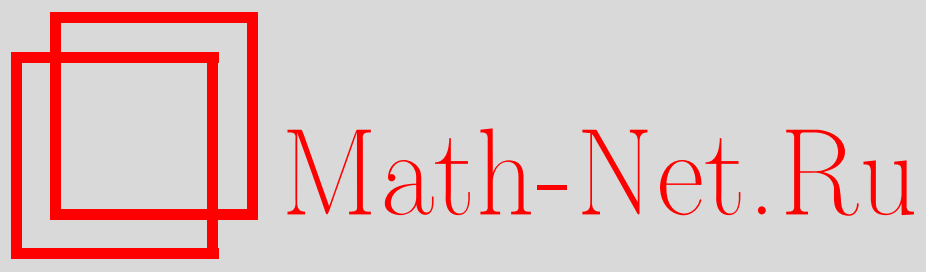

Ф. В. Петров, О количестве рациональных точек на строго выпуклой кривой, Функи. анализ и его прил., 2006, том 40, выпуск 1, 30-42

DOI: https://doi.org/10.4213/faa16

Использование Общероссийского математического портала Math-Net.Ru подразумевает, что вы прочитали и согласны с пользовательским соглашением

http://www . mathnet.ru/rus/agreement

Параметры загрузки:

IP : 52.87 .193 .239

26 апреля 2023 г., 11:30:15

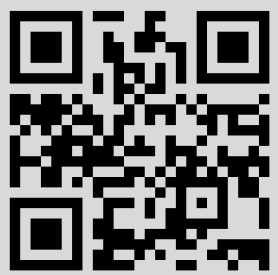


Функииональный анализ и его приложения

2006, т. 40, вып. 1, с. 30-42

УДК 511.9

\title{
О количестве рациональных точек на строго выпуклой кривой*
}

\author{
(C) 2006. Ф. В. ПЕтров
}

Введение

В знаменитой работе Ярника [1] было (в числе прочего) доказано, что максимальное количество целых точек, которые могут лежать на строго выпуклой кривой длины $N$ на плоскости, растет как $c N^{2 / 3}$ (точная константа $c$ также вычислена Ярником и равна $\left.3(2 \pi)^{-1 / 3}\right)$. Иными словами, количество точек сетки $L_{N}:=\left(\frac{1}{N} \mathbb{Z}\right)^{2}$, лежащих на строго выпуклой кривой $\gamma$ длины 1 , не превосходит $c N^{2 / 3}$ и для любого $N$ существует такая строго выпуклая кривая $\gamma^{(N)}$ длины 1 , чTо

$$
k\left(\gamma^{(N)}, N\right):=\#\left(\gamma^{(N)} \cap L_{N}\right) \geqslant c N^{2 / 3}+o\left(N^{2 / 3}\right) .
$$

В связи с этим возникает естественный вопрос: а существует ли универсальная кривая $\gamma$, для которой неравенство $(*)$ (возможно, с другим значением $c$ ) выполняется для бесконечного количества натуральных $N$ ? Этот вопрос сформулирован А. М. Вершиком, но впервые появляется в литературе, видимо, в работе Планя [2], в которой автор указывает, что был введен в проблематику Ж.-М. Дезуйе (J.-M. Deshouillers) и Дж. Грекосом (G. Grekos).

Свиннертон-Дайер [3] доказал, что если $\gamma \in C^{3}$, то $k(\gamma, N) \leqslant c N^{3 / 5+\varepsilon}$, а Бомбьери и Пила установили [4], что для бесконечно гладкой кривой $\gamma$ имеет место неравенство $k(\gamma, N) \leqslant c N^{1 / 2+\varepsilon}$ при любом $\varepsilon>0$. Мы здесь не будем перечислять многочисленные дальнейшие результаты в этом направлении, связанные с гладкостью, условиями на кривизну и другими ограничениями на кривую.

В работах Вершика и Барани [5,6] обсуждаются вопросы о предельных формах многоугольников с вершинами на мелкой сетке. Ответы на эти вопросы выявляют связь с аффинной геометрией. А именно, пусть $l_{a}(\gamma)$ обозначает аффинную длину кривой $\gamma$ (интеграл по натуральному параметру от кубического корня из кривизны). Оказывается, что количество многоугольников с вершинами в узлах сетки $L_{n}$, лежащих в малой окрестности данной кривой $\gamma$, растет как $e^{c \cdot l_{a}(\gamma) n^{2 / 3}}$, а количество вершин этих многоугольников - как $c \cdot l_{a}(\gamma) n^{2 / 3}$ (замечательно, что это верно как для максимально возможного количества вершин, так и для количества вершин типичного многоугольника, различаются только константы). Многоугольники с вершинами в узлах сетки $L_{n}$, содержащиеся в данном выпуклом многоугольнике, концентрируются около замкнутой выпуклой кривой, которая имеет максимально возможную аффинную длину [6]. Эта кривая составлена из кусков парабол, вписанных в углы многоугольника. Поэтому количество узлов сетки $L_{N}$ на такой кривой не превосходит $C N^{1 / 2}$. Таким

* Работа выполнена при поддержке грантов НШ.2251.2003.1, RUM1-2622-ST-04 (CRDF) и гранта РФФИ №05-01-00899. 
образом, типичная кривая не является универсальной. Здесь мы покажем, что в действительности универсальной кривой не существует. А именно, имеет место

ТЕОРема 1. Пусть $\gamma$ - ограниченная строго выпуклая кривая. Тогда $k(\gamma, n)$ $=o\left(n^{2 / 3}\right)$.

Я глубоко признателен А. М. Вершику за постановку задачи, многочисленные полезные обсуждения и внимание к работе, включающее и труд по редактированию рукописи. Я также благодарен А. Городнику за ценную консультацию по равномерной распределенности.

\section{§1. Определения и обозначения}

Зафиксируем на плоскости декартову систему координат.

Будем обозначать через $S(F)$ удвоенную площадь многоугольника $F$; через $\mathbf{x} \times \mathbf{y}$ будем обозначать псевдоскалярное произведение векторов $\mathbf{x}$ и $\mathbf{y}$ (т.е. ориентированную площадь параллелограмма, построенного на этих векторах).

Зафиксируем на плоскости треугольник $A B C$, ориентированный так, что $S=S(A B C)=+\overline{A C} \times \overline{C B}$. Введем следующие понятия:

1. $\mathrm{An}=\operatorname{An}(A B C)$ - угол с вершиной в начале координат, образованный лучами, сонаправленными с $A C$ и $C B$ (величина этого угла есть $\pi-\angle A C B$ ). Угол будем рассматривать как множество векторов $\mathbf{x} \in \mathbb{R}^{2}$, которые, будучи отложенными из начала координат, лежат в An.

2. Определим обхват $[\mathbf{x}]$ вектора $\mathbf{x}$ (относительно треугольника $A B C$ ) формулой

$$
[\mathbf{x}]=(\mathbf{x} \times \overline{C B}+\overline{A C} \times \mathbf{x}) / S .
$$

Обхват - линейная функция вектора $\mathbf{x} \in \mathbb{R}^{2}$. Заметим, что $[\overline{A C}]=[\overline{C B}]=1$, $[\overline{A B}]=2$. Будем называть обхватом отрезка $P Q$ величину $[P Q]=|[\overline{P Q}]|$.

3. Определим $A B C$-радиус произвольного треугольника как произведение обхватов его сторон, деленное на учетверенную площадь. (Если вместо обхватов брать длины сторон, получится радиус описанной окружности. В действительности $A B C$-радиус можно интерпретировать как «радиус» описанной около треугольника параболы с осью, параллельной вектору $\overline{A C}+\overline{B C}$.) Заметим, что $A B C$-радиус треугольника $A B C$ равен $S^{-1}$.

4. Строго выпуклую ломаную вида $A C_{1} C_{2} \ldots C_{k} B$ будем называть $(A B, C)-л o$ маной, если все ее вершины лежат в треугольнике $A B C$. Если при этом промежуточные вершины $C_{i}(i=1, \ldots, k)$ лежат на сетке $L_{n}=\left(\frac{1}{n} \mathbb{Z}\right)^{2}$, будем называть такую ломаную $(A B, C ; n)$-ломаной.

5. Пусть $(A B, C)$-ломаная $\gamma=A C_{1} C_{2} \ldots C_{k} B$ вписана в $(A B, C)$-ломаную $\gamma_{1}=A D_{1} D_{2} \ldots D_{k+1} B$ (т.е. точки $C_{i}$ лежат на соответствующих отрезках $\left.D_{i} D_{i+1}(i=1, \ldots, k)\right)$. Будем называть обобщенной аффинной длиной ломаной $\gamma$ относительно $\gamma_{1}$ величину

$$
l_{A}\left(\gamma: \gamma_{1}\right):=\sum_{i=0}^{k} S\left(C_{i} D_{i+1} C_{i+1}\right)^{1 / 3} \quad\left(C_{0}=A, C_{k+1}=B\right),
$$

а обобщенной аффинной длиной ломаной $\gamma$ величину

$$
l_{A}(\gamma)=\sup _{\gamma_{1}} l_{A}\left(\gamma: \gamma_{1}\right)
$$

где верхняя грань берется по всем $(A B, C)$-ломаным $\gamma_{1}$, описанным около $\gamma$. 


\section{§2. План доказательства теоремы 1 и предварительные утверждения}

Прежде всего нам понадобится оценка количества $k$ промежуточных вершин $(A B, C ; n)$-ломаной при больших $n$. Для этого воспользуемся тем известным фактом, что площадь выпуклого $k$-угольника с вершинами на сетке $\mathbb{Z}^{2}$ не меньше чем $\left(8 \pi^{2}\right)^{-1} k^{3} \geqslant(k / 5)^{3}(k \geqslant 3)$. Отсюда получаем, что

$$
k \leqslant \max \left(3,5\left(S n^{2}\right)^{1 / 3}\right) .
$$

Таким образом, максимальное количество узлов сетки $L_{n}$, лежащих в треугольнике площади $S$ и образующих выпуклый многоугольник, растет не быстpeе чем $c\left(S n^{2}\right)^{1 / 3}$. В действительности оно растет в точности как $c\left(S n^{2}\right)^{1 / 3}$. Пример $(A B, C ; n)$-ломаной с количеством звеньев порядка $c\left(S n^{2}\right)^{1 / 3}$ может быть построен следующим образом: упорядочим векторы множества $\mathrm{An} \cap L_{n}$ в порядке возрастания обхвата. Возьмем $c\left(S n^{2}\right)^{1 / 3}$ векторов с наименьшим обхватом и построим выпуклую ломаную, для которой эти векторы будут векторами звеньев. При не слишком большом $c$ (скажем, $c=1 / 100)$ эту ломаную можно параллельно перенести в треугольник $A B C$ так, чтобы ее вершины лежали в узлах сетки $L_{n}$ и вместе с вершинами $A$ и $B$ образовывали $(A B, C)$-ломаную. Приведенная конструкция аналогична конструкции Ярника [1] ломаной данной длины, содержащей наибольшее количество целых точек, и может служить ее «аффинным» обобщением. Этот пример понадобится лишь в §4.

Идея доказательства теоремы 1 такова: проводя касательные к кривой в точках ее пересечения с сеткой $L_{n}$, при больших $n$ получаем много маленьких треугольников, в объединении которых содержится наша кривая. Оказывается, что если точек на этих сетках достаточно много, то сумма кубических корней из площадей этих треугольников (величина, названная обобщенной аффинной длиной) будет при добавлении точек очередной сетки уменьшаться в некоторое постоянное число раз (лемма 4), что невозможно. Для доказательства леммы 4 используются некоторые технические утверждения, которым и посвящен этот параграф.

Рассмотрим все векторы из множества $\mathbb{Z}^{2} \cap$ An и упорядочим их по возрастанию обхвата. Рассмотрим $k$ таких векторов $\mathbf{z}_{1}, \ldots, \mathbf{z}_{k}$ с наименьшим обхватом.

Набор векторов $\mathbf{z}_{1}, \ldots, \mathbf{z}_{k}$ устроен следующим образом: это все векторы, концы которых лежат в треугольнике $O P Q=\operatorname{An} \cap\left\{\mathbf{z}:[\mathbf{z}]<r=\left[\mathbf{z}_{k}\right]\right\}$, и несколько векторов с обхватом, равным $r$. Имеем

$$
k=\frac{1}{2} S(O P Q)+o\left(r^{2}\right)=\frac{S}{2} r^{2}+o\left(r^{2}\right) .
$$

Кроме того,

$$
\begin{aligned}
\sum\left[\mathbf{z}_{i}\right] & =\int_{O P Q}[\mathbf{x}] d \mathbf{x}+o\left(r^{3}\right)=r^{-1} S^{-1} \int_{O P Q}(S(O P X)+S(O Q X)) d \mathbf{x}+o\left(r^{3}\right) \\
& =\frac{S}{3} r^{3}+o\left(r^{3}\right) .
\end{aligned}
$$

Отсюда следует, что

$$
\sum\left[\mathbf{z}_{i}\right] \geqslant c S^{-1 / 2} k^{3 / 2}+o\left(k^{3 / 2}\right), \quad c=2 \sqrt{2} / 3 .
$$

Эта оценка понадобится нам в дальнейшем. 
Следующая элементарная лемма является технической основой всего дальнейшего.

Лемма 1. Пусть точки $P, R$ въбранъ на сторонах $A C$ и $B C$ треугольника ABC соответственно, а точка $Q-$ на отрезке $P R$. Тогда

1) $S(A Q P)^{1 / 3}+S(B Q R)^{1 / 3} \leqslant S^{1 / 3}$.

Кроме того, существует функиия $\varepsilon_{1}(\varepsilon)$, убьвающая $\kappa$ нулю при $\varepsilon \rightarrow 0$, такая, что если Err $:=1-(S(A P Q) / S)^{1 / 3}-(S(B Q R) / S)^{1 / 3}<\varepsilon[A Q]$, mо

2) $[A P] /[P Q] \in\left(1-\varepsilon_{1}, 1+\varepsilon_{1}\right)$;

3) $A B C$-радиус $r(A Q P)$ треугольника $A Q P$ лежит в $S^{-1} \cdot\left(1-\varepsilon_{1}, 1+\varepsilon_{1}\right)$;

4) $[\mathbf{x}]_{A Q P} /[\mathbf{x}] \in(A P / A C)\left(1-\varepsilon_{1}, 1+\varepsilon_{1}\right)$ для любого вектора $\mathbf{x} \in \operatorname{An}(A Q P)$ (через $[\mathbf{x}]_{A Q P}$ обозначается $A Q P$-обхват вектора $\mathbf{x}$ ).

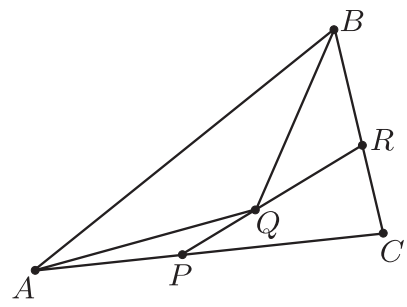

ДоказАтельство. Имеем

$$
\begin{aligned}
\operatorname{Err}= & 1-\left(\frac{S(A P Q)}{S}\right)^{1 / 3}-\left(\frac{S(B Q R)}{S}\right)^{1 / 3} \\
= & 1-\left(\frac{A P}{A C} \cdot \frac{P Q}{R P} \cdot \frac{R C}{C B}\right)^{1 / 3}-\left(\frac{P C}{A C} \cdot \frac{Q R}{R P} \cdot \frac{B R}{B C}\right)^{1 / 3} \\
= & \left(\frac{1}{3}\left(\frac{A P}{A C}+\frac{P Q}{R P}+\frac{R C}{C B}\right)-\left(\frac{A P}{A C} \cdot \frac{P Q}{R P} \cdot \frac{R C}{C B}\right)^{1 / 3}\right) \\
& +\left(\frac{1}{3}\left(\frac{P C}{A C}+\frac{Q R}{R P}+\frac{B R}{B C}\right)-\left(\frac{P C}{A C} \cdot \frac{Q R}{R P} \cdot \frac{B R}{B C}\right)^{1 / 3}\right)
\end{aligned}
$$

Выражения в двух последних скобках имеют вид $(x+y+z) / 3-(x y z)^{1 / 3}$ (и отсюда по неравенству о средних следует п. 1) леммы). Имеем

$$
\begin{aligned}
& 2(x+y+z)-6(x y z)^{1 / 3} \\
& \quad=\left(x^{1 / 3}+y^{1 / 3}+z^{1 / 3}\right)\left(\left(x^{1 / 3}-y^{1 / 3}\right)^{2}+\left(y^{1 / 3}-z^{1 / 3}\right)^{2}+\left(z^{1 / 3}-x^{1 / 3}\right)^{2}\right) .
\end{aligned}
$$

Значит, если $\max (x, y, z) \geqslant(1+\delta) \min (x, y, z)$, то $(x+y+z) / 3-(x y z)^{1 / 3} \geqslant$ $c(\delta)(x+y+z)$, где $c(\delta)$ - положительная убывающая к нулю при $\delta \rightarrow 0$ функция. Применяя это наблюдение к первому слагаемому в правой части формулы $(3)$ $(x=A P / A C, y=P Q / R P, z=R C / C B)$, получаем, что если Err $\leqslant \varepsilon[A Q]$, то, так как $[A Q]=[A P]+[P Q]=A P / A C+(P Q / P R) \cdot[P R] \leqslant(A P / A C)+2(P Q / P R)$, справедлива оценка $\operatorname{Err} \leqslant 2 \varepsilon(x+y+z)$. Поэтому $\max (x, y, z) / \min (x, y, z)<1+\varepsilon_{1}$, где $\varepsilon_{1}$ удовлетворяет условию $c\left(\varepsilon_{1}\right)>2 \varepsilon$. Это $\varepsilon_{1}(\varepsilon)$ можно выбрать так, что оно 
будет стремиться к нулю при $\varepsilon \rightarrow 0$. Далее,

$$
\begin{aligned}
{[P A] } & =x, \\
{[P Q]=\frac{P Q}{P R}[P R]=y([P C]+[C R]) } & =y(1-x+z)=[P A] \frac{y}{x}(1-x+z) .
\end{aligned}
$$

Имеем

$y / x \in\left(1-\varepsilon_{1}, 1+\varepsilon_{1}\right), \quad 1-x+z=1+z(1-x / z) \in\left(1-z \varepsilon_{1}, 1+z \varepsilon_{1}\right) \subset\left(1-\varepsilon_{1}, 1+\varepsilon_{1}\right) ;$ поэтому

$$
[P A] /[P Q] \in\left(\left(1-\varepsilon_{1}\right)^{2},\left(1+\varepsilon_{1}\right)^{2}\right) \subset\left(1-\varepsilon_{2}, 1+\varepsilon_{2}\right), \quad \varepsilon_{2}:=2 \varepsilon_{1}+\varepsilon_{1}^{2},
$$

что доказывает п. 2) (с $\varepsilon_{2}$ вместо $\left.\varepsilon_{1}\right)$.

Для доказательства п. 3) заметим, что

$$
\begin{aligned}
S \cdot r(A Q P) & =[A P][P Q] \frac{[A P]+[P Q]}{2} \frac{S}{S(A P Q)} \\
& =\frac{[A P][P Q]([A P]+[P Q])}{2 x y z} \in\left(1-\varepsilon_{3}, 1+\varepsilon_{3}\right),
\end{aligned}
$$

где $\varepsilon_{3}(\varepsilon) \rightarrow 0$ при $\varepsilon \rightarrow 0$, так как попарные отношения чисел $[A P]=x,[P Q]$, $y, z$ лежат в отрезке $\left(1-\varepsilon_{2}, 1+\varepsilon_{2}\right)$.

Перейдем к доказательству п. 4). Пусть $\mathbf{x}=a \overline{A P}+b \overline{P Q}, \mathbf{x} \in \operatorname{An}(A Q P)$ $(a, b \geqslant 0)$. Тогда $[\mathbf{x}]_{A Q P}=a+b,[\mathbf{x}]=a[A P]+b[P Q]=(a+b)[A P]+b([P Q]-$ $[A P])=(A P / A C)(a+b)(1+(b /(a+b))([P Q] /[A P]-1))$. Отсюда и следует п. 4).

Применяя п. 1) леммы 1 несколько раз, мы приходим к такому результату:

СлЕдСтвиЕ. Афбинная длина любой $(A B, C)$-ломаной $\gamma$ удовлетворяет неравенству $l_{A}(\gamma) \leqslant S^{1 / 3}$. Кроме того, афбинная длина не увеличивается при добавлении к ломаной новых вершин.

ЗАмЕЧАНиЕ. Как указал автору С. В. Дужин, п. 1) леммы 1 можно найти, например, в книге [7, с. 391].

Сейчас мы сформулируем утверждение об асимптотическом распределении целых точек на поверхности $\{a b-c d=$ const $\}$.

Лемма 2. Рассмотрим пары векторов $\left(\mathbf{x}_{1}, \mathbf{x}_{2}\right), \mathbf{x}_{1}, \mathbf{x}_{2} \in \mathrm{An} \cap \mathbb{Z}^{2}$, для котоpъх $\mathbf{x}_{1} \times \mathbf{x}_{2}=m$, где $0 \neq m \in \mathbb{Z}-$ некоторая константа ${ }^{1)}$. Кажной такой паре сопоставим специальную точку $\left(\left[\mathbf{x}_{1}\right],\left[\mathbf{x}_{2}\right]\right) \in[0, \infty)^{2}$. Тогда специальнье точки распределены равномерно в первом квадранте в следующем смысле: для любой ограниченной области $\Omega \subset(0, \infty)^{2}$ с кусочно-гладкой границей количество спеииальных точек в области $N \Omega$ ( с учетом кратности) есть (при $N \rightarrow \infty)$

$$
c(m) S N^{2} S(\Omega)+o\left(N^{2}\right),
$$

где $c(m)$ - константа, зависящая от $m\left(\right.$ а именно, $c(m)=(2 \zeta(2))^{-1} \sigma(m) / m$, $\sigma(m)$ - сумма натуральных делителей числа $m)$.

Доказательство леммы 2 вынесено в приложение. Как указал автору А. Городник, это утверждение можно вывести и из известных общих результатов (см., например, [8]).

1) Можно говорить и о $2 \times 2$-матрицах с целыми элементами и определителем $m$, однако в нашей ситуации будут фигурировать именно треугольники заданной площади. 
СлЕДСТВИЕ. Рассмотрим треугольники (определенные с точностью до параллельного переноса) $P Q R$, такие, что

1) $\overline{P Q}, \overline{Q R} \in \mathrm{An} \cap \mathbb{Z}^{2}$;

2) $[P R] \leqslant M(n / S)^{1 / 3}$;

3) $r(P Q R) \in 2 n S^{-1}\left(t_{1}, t_{2}\right)\left(0<t_{1}<t_{2}\right)$;

4) $S(P Q R) \leqslant m(m \in \mathbb{N})$.

Для числа $N\left(m, M, t_{1}, t_{2}\right)$ таких треугольников выполняется соотношение $($ nрu $n \rightarrow \infty)$

$$
N\left(m, M, t_{1}, t_{2}\right) \leqslant c(m)\left(t_{2}^{3}-t_{1}^{3}\right)\left(n S^{2}\right)^{1 / 3}+o\left(n^{2 / 3}\right) .
$$

ДокАЗАТЕльство сразу следует из леммы 2 для областей $\Omega\left(m^{\prime}, M, t_{1}, t_{2}\right)=$ $\left\{(p, q): 0<p, q<M, p q(p+q) \in 4 m^{\prime}\left(t_{1}, t_{2}\right)\right\}$ и числа $N=(n / S)^{1 / 3}\left(m^{\prime}=\right.$ $1, \ldots, m)$ и из того факта, что область $\Omega\left(m^{\prime}, \infty, 0,1\right)$ имеет конечную площадь.

ЛЕмма 3. Рассмотрим выпуклый четырехуголъник $P W T R$, такой, что $\overline{P W}, \overline{W T}, \overline{T R} \in$ An. Пусть $Q-$ точка на его стороне $W T$. Тогда число $r(P Q R)$ лежит между числами $([P Q] /[P W]) r(P Q W)$ u $([Q R] /[T R]) r(Q T R)$.

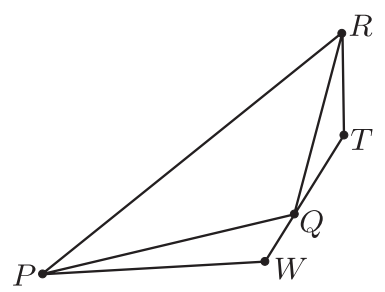

ДокАзАТЕЛЬСтво. Имеем

$$
S(P Q R)=([Q R] /[W Q]) S(P Q W)+([P Q] /[Q T]) S(R Q T) .
$$

Это равенство может показаться неожиданным: правая часть априори зависит от линейной функции $[\mathbf{x}]$.

Доказать его можно, например, так: при движении точек $W$ и $T$ по лучам $Q W$ и $Q T$ ни левая, ни правая части равенства не меняются. Поэтому можно считать, что $[W Q]=[Q R]$ и $[Q T]=[P Q]$. Первое равенство означает, что прямая, соединяющая $Q$ с серединой отрезка $R W$, параллельна вектору $\overline{A C}+\overline{B C}$. Аналогично, второе равенство означает, что этому вектору параллельна прямая, соединяющая $Q$ с серединой отрезка $P T$. Таким образом, точка $Q$ лежит на прямой Гаусса четырехугольника $P R T W$, а потому удовлетворяет задающему эту прямую равенству $S(P X R)+S(T X W)=S(W X P)+S(R X T)$ (разумеется, прямую задает равенство с ориентированными, а не обычными площадями), что и требовалось доказать. Отсюда получаем

$$
\begin{aligned}
2 r(P Q R) & =\frac{[P Q][Q R]([P Q]+[Q R])}{([Q R] /[W Q]) S(P Q W)+([P Q] /[Q T]) S(R Q T)} \\
& =\frac{[P Q]+[Q R]}{[P W] /(2 r(P Q W))+[T R] /(2 r(Q T R))} .
\end{aligned}
$$

Как известно, частное $(x+y) /\left(x^{\prime}+y^{\prime}\right)$ лежит между $x / x^{\prime}$ и $y / y^{\prime}$. Полагая $x=$ $[P Q], y=[Q R]$ и $x^{\prime}=[P W] / r(P Q W), y^{\prime}=[T R] / r(Q T R)$, получаем требуемое. 


\section{§3. Основная часть}

Лемма 4. Для любого $c>0$ существует такое число $a(c)>0$, что для любого треугольника $A B C$ при всех достаточно больших натуральных $n>$ $N(c, \triangle A B C)$ справедливо следующее утверждение: для любой $(A B, C ; n)$-ломаной $\gamma$ с количеством звенъев $\geqslant c\left(n^{2} S(A B C)\right)^{1 / 3}$ выполняется неравенство $l_{A}(\gamma) \leqslant(1-a(c)) S(A B C)^{1 / 3}$.

Иными словами, если в ломаной достаточно много звеньев, то у нее не слишком большая обобщенная аффинная длина.

Покажем, как из леммы 4 следует теорема 1.

Предположим, что теорема неверна и для некоторой ограниченной строго выпуклой кривой $\gamma$ выполняются неравенства $k\left(\gamma, q_{n}\right) \geqslant c q_{n}^{2 / 3}$ для некоторой возрастающей последовательности натуральных чисел $q_{1}<q_{2}<q_{3}<\cdots$. Не умаляя общности, можно считать, что кривая $\gamma$ соединяет точки $A$ и $B$, находясь внутри некоторого треугольника $A B C$ (любая ограниченная выпуклая кривая разбивается на конечное число таких кривых). Пусть также $S(A B C)=1$. Обозначим через $\gamma_{n}$ вписанную в $\gamma(A B, C)$-ломаную, содержащую в качестве промежуточных вершин все точки пересечения $\gamma \cap\left(\bigcup_{i=1}^{n} L_{q_{i}}\right)$. Зафиксируем в каждой точке кривой $\gamma$ опорную прямую к $\gamma$. Проведя эти прямые в вершинах кривой $\gamma_{n}$, получим $(A B, C)$-ломаную $\gamma_{n}^{\prime}$, описанную около $\gamma_{n}$. При пересечении опорных прямых в соседних вершинах ломаной $\gamma_{n}$ образуются треугольники $\triangle_{1}, \ldots, \triangle_{k}$. Кривая $\gamma$ лежит в объединении $\bigcup_{i=1}^{k} \triangle_{i}$. Положим $S_{i}=S\left(\triangle_{i}\right)$. Пусть $q=q_{m}$ - такое большое натуральное число, что для каждого треугольника $\triangle_{i}(1=1, \ldots, k)$ выполняется альтернатива из леммы 4: либо

(1) $\#\left(\gamma \cap \triangle_{i} \cap L_{q}\right) \leqslant(c / 2) S_{i}^{1 / 3} q^{2 / 3}$, либо

(2) $l_{A}\left(\gamma_{m} \cap \triangle_{i}\right) \leqslant(1-a) S_{i}^{1 / 3}$, где $a=a(c / 2)$ - число, зависящее только от $c$.

Обозначим через $M_{1}$ множество номеров $i$, для которых имеет место случай (1), а через $M_{2}$ - множество остальных номеров $i$ (для них имеет место случай $(2))$. Пользуясь следствием из леммы 1 и неравенством (1), получаем

$$
\begin{aligned}
c\left(\sum_{i=1}^{k} S_{i}^{1 / 3}\right) q^{2 / 3} & \leqslant c S(A B C)^{1 / 3} q^{2 / 3}=c q^{2 / 3} \leqslant \#\left(\gamma \cap L_{q}\right) \leqslant \sum_{i=1}^{k} \#\left(\gamma \cap L_{q} \cap \triangle_{i}\right) \\
& =\sum_{i \in M_{1}} \#\left(\gamma \cap L_{q} \cap \triangle_{i}\right)+\sum_{i \in M_{2}} \#\left(\gamma \cap L_{q} \cap \triangle_{i}\right) \\
& \leqslant \frac{c}{2}\left(\sum_{i \in M_{1}} S_{i}^{1 / 3}\right) q^{2 / 3}+5\left(\sum_{i \in M_{2}} S_{i}^{1 / 3}\right) q^{2 / 3} \\
& \leqslant \frac{c}{2}\left(\sum_{i=1}^{k} S_{i}^{1 / 3}\right) q^{2 / 3}+5\left(\sum_{i \in M_{2}} S_{i}^{1 / 3}\right) q^{2 / 3},
\end{aligned}
$$

откуда вытекает, что

$$
\sum_{i \in M_{2}} S_{i}^{1 / 3} \geqslant \frac{c}{10} \sum_{i=1}^{k} S_{i}^{1 / 3} .
$$


Далее,

$$
\begin{aligned}
l_{A}\left(\gamma_{m}: \gamma_{m}^{\prime}\right) & \leqslant \sum_{i=1}^{k} l_{A}\left(\gamma_{m} \cap \triangle_{i}\right)=\sum_{i \in M_{1}}+\sum_{i \in M_{2}} \leqslant \sum_{i \in M_{1}} S_{i}^{1 / 3}+(1-a) \sum_{i \in M_{2}} S_{i}^{1 / 3} \\
& \leqslant\left(1-\frac{a c}{10}\right) \sum_{i=1}^{k} S_{i}^{1 / 3}=\left(1-\frac{a c}{10}\right) l_{A}\left(\gamma_{n}: \gamma_{n}^{\prime}\right) .
\end{aligned}
$$

Отсюда следует, что $\lim _{n \rightarrow \infty} l_{A}\left(\gamma_{n}: \gamma_{n}^{\prime}\right)=0$, а это противоречит сделанным предположениям (из которых вытекает, что $\left.l_{A}\left(\gamma_{n}: \gamma_{n}^{\prime}\right) \geqslant c / 5\right)$ ).

Таким образом, достаточно доказать лемму 4.

Пусть $\gamma=C_{0} C_{1} C_{2} \ldots C_{k} C_{k+1}$ (при этом $C_{0}=A, C_{k+1}=B, C_{i} \in L_{n}, i=$ $1, \ldots, k)$ есть $(A B, C ; n)$-ломаная и $k \geqslant c S^{1 / 3} n^{2 / 3}$ (здесь и далее $S=S(A B C)$ уже не обязательно равно 1). Зафиксируем опорные прямые $l_{i}$ в точках $C_{i}(i=$ $1, \ldots, k)$, и пусть $l_{0}=A C$ и $l_{k+1}=B C$. Положим $l_{i} \cap A C=B_{i}(i=1, \ldots, k+1)$, $l_{i} \cap l_{i+1}=D_{i}(i=0,1, \ldots, k)$.

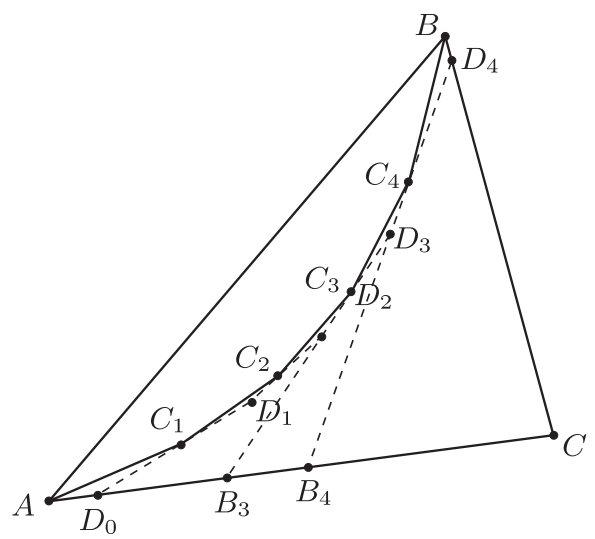

Имеем

$$
l_{A}(\gamma)=\sup \sum_{i=0}^{k} S\left(C_{i} D_{i} C_{i+1}\right)^{1 / 3},
$$

где верхняя грань берется по всем способам выбора опорных прямых к ломаной $\gamma$. Далее,

$$
\begin{gathered}
S(A B C)^{1 / 3}-l_{A}(\gamma)=\sup \sum_{i=1}^{k} x_{i} \\
x_{i}=S\left(A C_{i+1} B_{i+1}\right)^{1 / 3}-S\left(C_{i} C_{i+1} D_{i}\right)^{1 / 3}-S\left(A C_{i} B_{i}\right)^{1 / 3} .
\end{gathered}
$$

Числа $x_{i}$ неотрицательны согласно п. 1$)$ леммы 1 . Наша цель - найти $N \geqslant$ $c_{1} n^{2 / 3} S(A B C)^{1 / 3}$ номеров $i$, для которых $x_{i} \geqslant c_{2}\left[C_{i} C_{i+1}\right] S(A B C)^{1 / 3}$ (с некоторыми константами $c_{1}$ и $c_{2}$, зависящими от $\left.c\right)$. Если нам это удастся, лемма 4 будет доказана в силу оценки (2) для суммы $N$ минимально возможных обхватов. 
Отметим, что если мы найдем $N_{1} \geqslant c_{3} n^{2 / 3} S(A B C)^{1 / 3}$ номеров $i$, для которых $x_{i} \geqslant c_{4}\left[C_{i} C_{i+1}\right] S\left(A C_{i+1} B_{i+1}\right)^{1 / 3}$, то цель также будет достигнута. Действительно, в силу неравенства (1) справедлива оценка $100 n^{2} S\left(A C_{i+1} B_{i+1}\right) \geqslant i^{3}$, так что при $i \geqslant N_{1} / 2$ имеем $S\left(A C_{i+1} B_{i+1}\right)^{1 / 3} \geqslant c_{6} S(A B C)^{1 / 3} ;$ значит, для хотя бы $N_{1} / 2$ номеров $i$ будет выполнена требуемая оценка $x_{i} \geqslant c_{7}\left[C_{i} C_{i+1}\right] S(A B C)^{1 / 3}$.

Как следует из леммы 1 , примененной сначала к треугольнику $A B C$ и точкам $P=B_{i+1}, Q=C_{i+1}$, а затем к треугольнику $A C_{i+1} B_{i+1}$ и точкам $P=B_{i}$, $Q=C_{i}$, достаточно для некоторого $\varepsilon_{0}(c)$ найти $N_{1} \geqslant c_{3} n^{2 / 3} S(A B C)^{1 / 3}$ номеров $i$, для которых $\max \left(\left[C_{i} D_{i}\right],\left[D_{i} C_{i+1}\right]\right) / \min \left(\left[C_{i} D_{i}\right],\left[D_{i} C_{i+1}\right]\right)>1+\varepsilon_{0}$ или $r\left(C_{i} D_{i} C_{i+1}\right) \notin S^{-1}\left(1-\varepsilon_{0}, 1+\varepsilon_{0}\right)$. Назовем номер $i$, удовлетворяющий одному из этих условий, $\varepsilon_{0}$-выделяющимся. Из леммы 3 (для $P W T R=C_{i} D_{i} D_{i+1} C_{i+2}$, $\left.Q=C_{i+1}\right)$ вытекает такое

ПРЕДЛОЖЕНИЕ. Если номера $i$ u $i+1$ не являются $\varepsilon_{0}$-въделяюшимися, то для некоторого $\varepsilon_{1}\left(\varepsilon_{0}\right)$ выполнено соотношение $r\left(C_{i} C_{i+1} C_{i+2}\right) \in 2 S^{-1}\left(1-\varepsilon_{1}, 1+\right.$ $\left.\varepsilon_{1}\right)$, причем $\varepsilon_{1}$ убывает $к$ нулю вместе с $\varepsilon_{0}$.

Таким образом, достаточно доказать, что при $\varepsilon>0$ количество $N_{\varepsilon}$ номеров $i$, для которых $r\left(C_{i} C_{i+1} C_{i+2}\right) \in 2 S^{-1}(1-\varepsilon, 1+\varepsilon)$, допускает оценку сверху $N_{\varepsilon} \leqslant \varepsilon_{1}\left(S n^{2}\right)^{1 / 3}$, где $\varepsilon_{1}$ стремится к нулю вместе с $\varepsilon$.

Заметим, что $\sum S\left(C_{i} C_{i+1} C_{i+2}\right)^{1 / 3} \leqslant 2 S^{1 / 3}$ (суммы по четным и нечетным $i$ не превосходят $S^{1 / 3}$ по следствию из леммы 1$)$, так что количество номеров $i$, для которых $S\left(C_{i} C_{i+1} C_{i+2}\right) \geqslant m n^{-2}$, не превосходит (по неравенству Чебышёва) $2 m^{-1 / 3}\left(S n^{2}\right)^{1 / 3}$. Кроме того, так как сумма обхватов $\sum_{i=0}^{k}\left[C_{i} C_{i+1}\right]$ меньше или равна 2, мы также можем, не умаляя общности, считать (опять применяя неравенство Чебышёва), что $\left[C_{i} C_{i+2}\right] \leqslant M\left(n^{2} S\right)^{-1 / 3}$.

Осталось воспользоваться следствием из леммы 2.

ЗАмЕчАнИЕ. Утверждение теоремы можно несколько усилить, полагая $L_{n}=$ $\left(\frac{1}{n} \mathbb{Z}\right)^{2}+\mathbf{x}_{n}$, где $\mathbf{x}_{n}$ - некоторый вектор смещения сетки, и выбирая $n$ не обязательно целым.

\section{§4. О возможном количестве точек на выпуклой кривой}

В работе [2] был приведен пример строго выпуклой ограниченной кривой $\gamma$, для которой $k\left(\gamma, q_{n}\right) \geqslant c_{n} q_{n}^{2 / 3}$, где $q_{1}<q_{2}<\cdots$ - сколь угодно быстро растущая последовательность натуральных чисел, а коэффициенты $c_{n}$ стремятся к нулю экспоненциально $\left(c_{n}=K^{-n}, K>1\right.$ - некоторая явно указываемая константа). Здесь мы улучшим этот результат, показав, что достаточно сходимости ряда $\sum c_{n}$. Однако наш метод является довольно грубым, и кажется вероятным, что и это условие может быть ослаблено (возможно, до необходимого условия $\left.\lim c_{n}=0\right)$.

TEOPEMA 2. Пусть $\sum c_{k}<\infty\left(c_{i}>0\right)$ u $M \subset \mathbb{N}-$ бесконечное множество натуральных чисел. Тогда существует последовательность $q_{1}<q_{2}<\cdots, q_{i} \in M$, и ограниченная строго выпуклая кривая $\gamma$, такие, что $k\left(\gamma, q_{n}\right) \geqslant c_{n} q_{n}^{2 / 3}$.

Доказательство. Рассмотрим полуокружность длины $\sum c_{i}$. Разделим ее на дуги длин $c_{i}$, и пусть концы дуги длины $c_{i}$ есть $A_{i}$ и $A_{i+1}$. Проведем в концах дуг касательные к окружности. Каждая дуга $c_{i}$ попадет в один из возникающих при этом треугольников $A_{i} B_{i} A_{i+1}$, у каждого из которых две стороны лежат на 
касательных, а одна является хордой $A_{i} A_{i+1}$. Площадь треугольника $A_{i} B_{i} A_{i+1}$ не меньше, чем $C c_{i}^{3}$, где $C$ не зависит от $i$. Не умаляя общности, можно считать, что $C>100$ (в противном случае мы применим гомотетию с подходящим коэффициентом, увеличивая площади треугольников в $100 / C$ раз). Теперь построим в треугольнике $A_{i} B_{i} A_{i+1}\left(A_{i} A_{i+1}, B ; q_{i}\right)$-ломаную с вершинами в узлах $L_{q_{i}}$, где $q_{i} \in M$ - такое большое число, что количество промежуточных вершин этой ломаной больше или равно $c_{i} q_{i}^{2 / 3}$ (возможность построения такой ломаной обсуждалась в §1). Объединение всех таких ломаных даст требуемую кривую $\gamma$ (строго говоря, следует заменить прямые звенья ломаных на близкие строго выпуклые кривые, чтобы кривая $\gamma$ стала строго выпуклой).

\section{Приложение. О распределении целых точек на поверхности $\{a b-c d=$ const $\}$}

Рассмотрим ограниченные квадрируемые области $\Omega_{1}, \Omega_{2} \subset \mathbb{R}^{2}$. Поставим следующий вопрос: какова асимптотика количества $M\left(\Omega_{1}, \Omega_{2} ; n\right)$ пар векторов $\mathbf{x}_{1} \in n \Omega_{1} \cap \mathbb{Z}^{2}, \mathbf{x}_{2} \in n \Omega_{2} \cap \mathbb{Z}^{2}$, таких, что $\mathbf{x}_{1} \times \mathbf{x}_{2}=1$. Рассмотрим сначала случай треугольников

$$
\Omega_{i}=\left\{(x, y): 0<y<x \leqslant a_{i}\right\} \quad\left(i=1,2, a_{i}>0\right) .
$$

Тогда задача сводится к поиску числа решений неравенства

$$
x_{1} y_{2}-y_{1} x_{2}=1
$$

при условии, что

$$
0<y_{1}<x_{1}<n a_{1}, \quad 0<y_{2}<x_{2}<n a_{2} .
$$

Хорошо известно, что при фиксированных взаимно простых $x_{1}, x_{2}$ уравнение (5) имеет единственное решение $y_{1}, y_{2}$ при условии, что $0<y_{1} \leqslant x_{1}$, $0<y_{2} \leqslant x_{2}$. Случаи равенства $y_{1}=x_{1}$ или $y_{2}=x_{2}$ имеют место только для $x_{1}=1$ или $x_{2}=1$, т. е. для не более чем $C\left(a_{1}, a_{2}\right) n$ вариантов. Поскольку количество точек с взаимно простыми координатами $\left(x_{1}, x_{2}\right)$ в прямоугольнике $0<x_{1}<n a_{1}, 0<x_{2}<n a_{2}$ есть

$$
\zeta(2)^{-1} n^{2} a_{1} a_{2}+o\left(n^{2}\right)
$$

получаем в случае (4) асимптотику

$$
M\left(\Omega_{1}, \Omega_{2} ; n\right)=\zeta(2)^{-1} n^{2} a_{1} a_{2}+o\left(n^{2}\right) .
$$

Для дальнейших целей мы перепишем последний ответ следующим образом: если через $l(\Omega, \varphi)$ обозначить (одномерную лебегову) меру пересечения области $\Omega$ с прямой $y=\operatorname{tg} \varphi \cdot x$, идущей под углом $\varphi$ к оси абсцисс, то

$$
M\left(\Omega_{1}, \Omega_{2} ; n\right)=\zeta(2)^{-1} \int_{0}^{\pi} l\left(\Omega_{1}, \varphi\right) l\left(\Omega_{2}, \varphi\right) d \varphi \cdot n^{2}+o\left(n^{2}\right)
$$

(в случае (4) интеграл в (7) можно было бы брать и по меньшему отрезку).

Назовем базовыми треугольники площади $1 / 2$ вида $O A B$, где $O$ - начало координат, $A, B$ - целые точки.

Заметим, что формула (7) выполнена также для областей, получающихся из (4) аффинными действиями элементов группы $S L(2, \mathbb{Z})$. Иными словами, формула (7) верна для пар треугольников, получающихся из некоторого базового треугольника гомотетиями. 
Наш дальнейший план состоит в аппроксимации областей достаточно общего вида объединениями таких «базовых» областей, мало пересекающихся радиально (т. е. при центральной проекции на единичную окружность).

Зафиксируем число $\varepsilon>0$. Будем называть базовый треугольник $O A B$ $\varepsilon$-подходящим, если

1) $|O A / O B-1|<\varepsilon$

2) $\angle A O B<\varepsilon$.

Нам понадобится следующая

ЛЕмма 5. Почти любой (в смысле меры Лебега на единичной окружности) луч, выходящий из начала координат, идет внутрь бесконечного числа в-подходящих базовых треугольников.

ДокАЗАТЕЛЬСтво. Не умаляя общности, можно считать, что луч имеет вид $0<y=\alpha x, 0<\alpha<1$. Разложим число $\alpha$ в цепную дробь:

$$
\alpha=\frac{1}{a_{1}+\frac{1}{a_{2}+\cdots}} .
$$

Для почти всех $\alpha$ элементы $a_{i}$ не ограничены. (Известно и распределение элементов цепной дроби для почти всех $\alpha$ - формула Гаусса-Кузьмина. Используемый факт грубее и значительно проще доказывается. См. [9].) В терминах подходящих дробей $p_{k} / q_{k}(k=1,2, \ldots)$ это означает, что отношение соседних знаменателей $q_{k+1} / q_{k}$ не ограничено (так как $q_{k+1}=a_{k} q_{k}+q_{k-1}$ ). Каждой паре соседних подходящих дробей соответствует базовый треугольник $O A B$ $\left(A=\left(q_{k}, p_{k}\right), B=\left(q_{k+1}, p_{k+1}\right)\right)$, внутрь которого направлен луч $y=\alpha x$. Если отношение $q_{k+1} / q_{k}$ велико, то $O B \gg O A$.

Применим процедуру «вытягивания носов». А именно, построим последовательность точек $B_{0}=B, \overline{O B_{i}}=\overline{O B_{i-1}}+\overline{O A}$. Один из отрезков $B_{i-1} B_{i}$ пересечет наш луч. Треугольник $O B_{i-1} B_{i}$ будет базовым (так как по известному свойству подходящих дробей базовым был треугольник $O A B)$ и, если $a_{k}$ и $k$ достаточно велики, $\varepsilon$-подходящим. Лемма доказана.

Пусть теперь области $\Omega_{1}, \Omega_{2}$ суть гомотетичные треугольники вида $\Omega_{1}=$ $O C D, \Omega_{2}=\lambda \Omega_{1}(\lambda>0)$, где прямая $C D$ вертикальна и точки $C, D$ лежат в области $0<y<x(C$ ниже $D)$.

Согласно теореме Витали и лемме 5 , мы можем найти $\varepsilon$-подходящие треугольники $O A_{i} B_{i}(i=1, \ldots, n)$, такие, что лучи $O C, O A_{1}, O B_{1}, O A_{2}, O B_{2}, \ldots, O A_{n}$, $O B_{n}, O D$ идут против часовой стрелки в указанном порядке и $\sum_{i=1}^{n} \angle A_{i} O B_{i}>$ $\angle C O D-\varepsilon$.

Пусть $\Delta_{i}$ - наибольший треугольник, гомотетичный $O A_{i} B_{i}$, содержащийся в $\Omega_{1}(i=1, \ldots, n)$.

Тогда, суммируя оценки вида (7) для треугольников $\Delta_{i}, \lambda \Delta_{i}$, получаем оценку снизу

$$
M\left(\Omega_{1}, \Omega_{2} ; n\right) \geqslant\left(\lambda \sum_{i=1}^{n} 2 S\left(\Delta_{i}\right)\right) n^{2}+o\left(n^{2}\right) .
$$

При малом $\varepsilon$ имеет место оценка $S\left(\Delta_{i}\right) \geqslant c(\varepsilon) S\left(\angle A_{i} O B_{i} \cap \triangle O C D\right)$, где $c(\varepsilon) \rightarrow 1$ при $\varepsilon \rightarrow 0$. 
Таким образом, устремляя $\varepsilon$ к нулю, для указанных областей $\Omega_{1}, \Omega_{2}$ получаем оценку снизу вида (7)

$$
M\left(\Omega_{1}, \Omega_{2} ; n\right) \geqslant \zeta(2)^{-1} \int_{0}^{\pi} l\left(\Omega_{1}, \varphi\right) l\left(\Omega_{2}, \varphi\right) d \varphi \cdot n^{2}+o\left(n^{2}\right) .
$$

Рассмотрим точки $C_{1}$ и $D_{1}$, получающиеся пересечением прямой $C D$ с осью абсцисс и прямой $x=y$ соответственно. Рассмотрим треугольники $\triangle_{1}=O C_{1} C$, $\triangle_{2}=O C D, \triangle_{3}=O D D_{1}, \triangle_{0}=O C_{1} D_{1}$ и треугольники $\lambda_{i}$. Имеем

$$
M\left(\triangle_{0}, \lambda \triangle_{0} ; n\right) \leqslant \sum_{i=1}^{3} \sum_{j=1}^{3} M\left(\triangle_{i}, \lambda \triangle_{j} ; n\right) .
$$

Левая часть неравенства имеет асимптотику вида (7), а три слагаемых правой части (для которых $i=j$ ) - оценку снизу (8). Отсюда получаем, что оценка снизу во всех трех случаях является и оценкой сверху, а перекрестные члены дают вклад $о\left(n^{2}\right)$ (последнее и так ясно).

Тем самым асимптотика (7) установлена для областей указанного вида.

Теперь любые две «достаточно хорошие» (например, квадрируемые) области можно аппроксимировать снизу и сверху суммами и разностями таких областей (суммами трапеций с вертикальными сторонами).

Рассмотрим теперь несколько более общую задачу. А именно, заменим условие $\mathbf{x}_{1} \times \mathbf{x}_{2}=1$, налагаемое на пару векторов, условием $\mathbf{x}_{1} \times \mathbf{x}_{2}=m=$ const $\neq 0$. Снова, рассматривая сначала треугольники

$$
\Omega_{i}=\left\{(x, y): 0<y<x \leqslant a_{i}\right\} \quad\left(i=1,2, a_{i}>0\right),
$$

находим ответ в этом частном случае. В самом деле, если зафиксировать НОД $\left(x_{1}, x_{2}\right)=d\left(x_{1}, x_{2}\right.$ - абсциссы векторов $\left.\mathbf{x}_{1}, \mathbf{x}_{2}\right)$, то получим

$$
d^{-1} \zeta(2)^{-1} n^{2} a_{1} a_{2}+o\left(n^{2}\right)
$$

искомых пар. Суммируя по всем делителям числа $m$, получаем в этом случае асимптотику

$$
M\left(\Omega_{1}, \Omega_{2} ; m ; n\right)=\sigma(m)|m|^{-1} \zeta(2)^{-1} \int_{0}^{\pi} l\left(\Omega_{1}, \varphi\right) l\left(\Omega_{2}, \varphi\right) d \varphi \cdot n^{2}+o\left(n^{2}\right) .
$$

Обобщение на случай областей $\Omega_{1}, \Omega_{2}$ общего вида производится точно так же, как и для $m=1$.

Покажем теперь, как из доказанного утверждения следует лемма 2.

ДокАЗАТЕЛЬСтво лЕммы 2. Не умаляя общности, можно считать область $\Omega$ прямоугольником вида $\{0<x<A, 0<y<B\}$. В этом случае количество специальных точек в прямоугольнике $N \Omega$ есть количество пар векторов $(\mathbf{x}, \mathbf{y})$, $\mathbf{x} \times \mathbf{y}=m$, таких, что $\mathbf{x} \in N \Omega_{x}, \mathbf{y} \in N \Omega_{y}$, где $\Omega_{x}$ и $\Omega_{y}$ - области, определенные формулами

$$
\Omega_{x}=\{\mathbf{x} \in \operatorname{An}:[\mathbf{x}] \leqslant A\}, \quad \Omega_{y}=\{\mathbf{y} \in \operatorname{An}:[\mathbf{x}] \leqslant B\} .
$$

Применяя к этому частному случаю асимптотику вида $\left(7^{\prime}\right)$, получаем требуемoe. 


\section{ЛИТЕРАТУРА}

1. Jarnik $V$. Über die Gitterpunkte auf konvexen Kurven. Math. Z., 24, 500-518 (1926).

2. Plagne A. A uniform version of Jarník's theorem. Acta Arith., 87, No. 3, 255-267 (1999).

3. Swinnerton-Dyer H. P. F. The number of lattice points on a convex curve. J. Number Theory, 6, 128-135 (1974).

4. Bombieri E., Pila J. The number of integral points on arcs and ovals. Duke Math. J., 59, 337-357 (1989).

5. Вершик A. M. Предельная форма выпуклых целочисленных многоугольников и близкие вопросы. Функц. анализ и его прил., вып. 1, 28, 16-25 (1994).

6. Barany $I$. The limit shape of convex lattice polygons. Discrete Comput. Geom., 13, Nos. 3-4, 279-295 (1995).

7. Фавар Ж. Курс локальной дифференциальной геометрии. ИЛ, М., 1960.

8. Eskin A., McMullen C. Mixing, counting and equidistribution in Lie groups. Duke Math. J., 71, No. 1, 181-209 (1993).

9. Хинчин А. Я. Цепные дроби. Физматгиз, М., 1961.

С.-Петербургское отделение Математического института им. В. А. Стеклова

Поступило в редакцию e-mail: fedorpetrov@mail.ru 